\title{
Experimental Comparison of Carrier and Space Vector PWM Control Methods for Three-Phase NPC Converters
}

\author{
Ivo Pereira ${ }^{1}$, António Martins $^{2}$ \\ ${ }^{1,2}$ Faculty of Engineering of the University of Porto \\ Department of Electrical and Computer Engineering \\ Rua Dr Roberto Frias, s/n, 4200-465, Porto \\ Phone: +351225081816, e-mail: ee03113@fe.up.pt, ajm@fe.up.pt
}

\begin{abstract}
Multilevel converters are powerful converters in the medium-voltage high-power range. Inside this family of converters, the three-level neutral point clamped converter has its own importance. The main control methods associated with this converter are the carrier-based PWM with added DC components and the space vector PWM. Two of the most important features of the NPC converter are the neutral point balance and the output voltage spectrum. Both control methods efficiently manage the two characteristics. This paper experimentally compares these two issues and shows that the two methods produce almost the same results; the choice between one and the other should be made according to other requirements.
\end{abstract}

\section{Keywords}

Neutral point voltage, Pulse width modulation, Space vector modulation, Three-level converter, Voltage spectrum

\section{Introduction}

The smaller voltage steps created by a multilevel converter lead to the production of higher power quality waveforms and also reduce the $d v / d t$ stresses on the load, especially in AC motors, and reduce the electromagnetic compatibility problems [1].

Some applications for these relatively new converters include industrial drives, [2], Flexible AC Transmission Systems (FACTS), [3], and vehicle propulsion. One area where multilevel converters are particularly suitable is in medium-voltage drives. Although the main applications of multilevel converters are related with power conversion from DC to AC voltages (inverter operation), they are now being also applied in rectifier operation, namely interfacing renewable energy sources with the grid, [4], and interfacing high power drives in electric traction [1].
The most common multilevel converter topologies are the diode-clamped, the flying capacitor, and the cascaded H-bridge converter, [5].

The diode-clamped multilevel converter employs clamping diodes and series DC capacitors to produce AC voltage waveforms with multiple levels, [1], [6]. The converter can be generally configured as a multilevel topology, but only the three-level converter, also referred as Neutral Point Clamped (NPC) converter, has found wide application in medium-voltage high-power applications [1]-[2].

The main features of the NPC converter include reduced $d v / d t$ and Total Harmonic Distortion (THD) in its AC output voltages in comparison to the conventional twolevel converter. As in any multilevel converter it can be used in the medium-voltage applications to reach a certain voltage level without series connection of power semiconductors.

The three-level NPC converter is the most widely used topology, which is offered by several drive manufacturers, [5]. However, all of the three topologies have specific advantages and disadvantages, [5].

In this paper it is analyzed the main operating characteristics of the NPC converter, schematically represented in Fig. 1, namely its operation under carrierbased PWM control and space vector control with emphasis in the output voltage spectrum and the neutral point voltage control.

\section{PWM Control of the Three-Phase NPC Converter}

In a conventional two-level converter, carrier PWM techniques control each phase leg of the converter separately and the line-to-line voltage is a result of the phase legs voltage. 
Several different two-level carrier-based PWM techniques have been extended for controlling multilevel converters. The most used and easiest technique to implement uses several triangle carrier signals and one modulation signal per phase, [7]-[8].

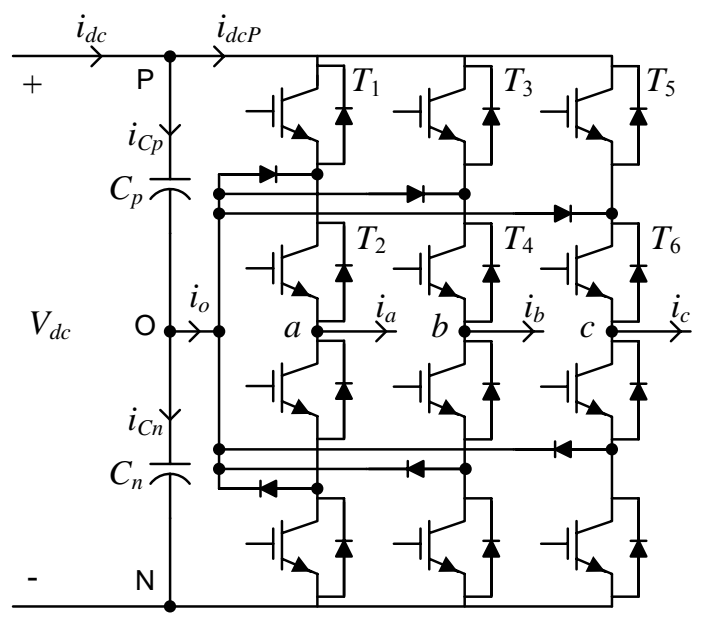

Fig. 1. Three-phase three-level NPC converter.

For multilevel converters, it is generally accepted that the phase disposition method gives rise to the lowest harmonic distortion [8]. This method was first applied to an $N$-level diode clamped converter, and arranges $N-1$ phase matched triangular carrier waveforms to fully occupy contiguous bands inside the normalized range between +1 and -1 . A single reference waveform for each phase is then compared against these carriers to determine how the phase leg should switch. Phase disposition modulation can be applied to cascaded converter structures to achieve the same harmonic performance. In contrast, Space Vector Pulse Width Modulation (SVPWM) identifies each switching state of a two- or multilevel converter as a point in complex space $(\alpha, \beta)$. Then a reference phasor rotating in the plane $(\alpha, \beta)$ at the fundamental frequency is sampled within each switching period, and the nearest three converter switched states are selected with duty cycles calculated to achieve the same volt-second average as the sampled reference phasor, [8]. This directly controls the converter line-to-line voltages, and only implicitly develops the phase leg voltages. The two-level space vector PWM technique can be extended to more than three levels for the diode-clamped converter.

Space vector and carrier modulation of two-level converters produce identical space vector sequences despite their apparent differences, [8]. The same is essentially true for multilevel converters: the optimized SVPWM switching sequences for a multilevel converter are the same as those created by carrier phase disposition modulation when an appropriate zero sequence component is injected into the carrier system references.

If working with three phase reference voltages instead of a voltage space vector, it is not possible to utilize the whole output range when the phase reference voltage system does not contain a zero sequence voltage component. The whole range may be utilized, if the zero sequence voltage component is added to the three phase reference voltages that make the absolute values of the maximum and minimum phase reference voltage equal to each other, [9].

In order to achieve a better DC link voltage utilization at high modulation indices the sinusoidal reference signal can be injected by a third harmonic with a magnitude equal to $25 \%$ of the fundamental. The DC utilization of Third Harmonic injection PWM (THPWM) and SVPWM are better than Sinusoidal PWM (SPWM) in the linear modulation region, [8], [10].

So, the three major carrier-based techniques used in a conventional two-level converter that can be applied in a multilevel converter are: sinusoidal PWM, third harmonic injection PWM, and space vector PWM. Their representation is shown in Fig. 2.

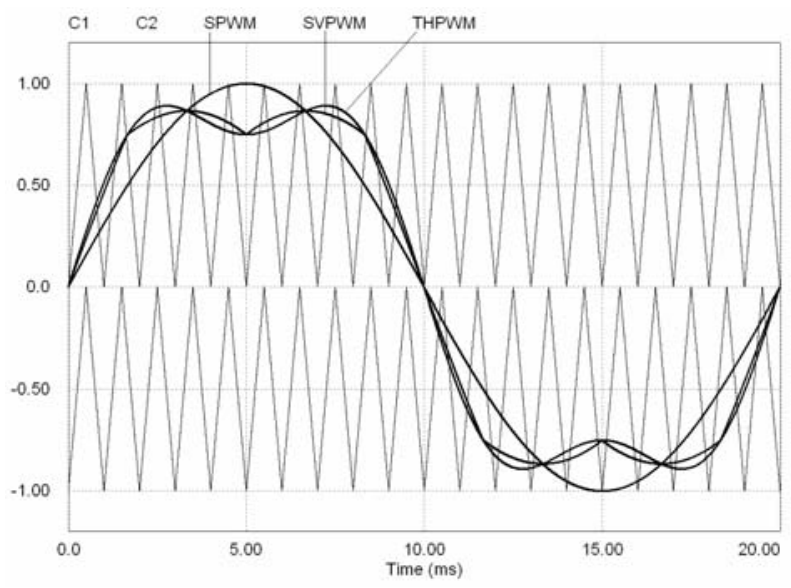

Fig. 2. The three main PWM techniques at unity modulation index, $50 \mathrm{~Hz}$ output frequency and $1 \mathrm{kHz}$ switching frequency: SPWM, THPWM, and SVPWM.

The two techniques, carrier and space vector modulation, are the most important methods for controlling multilevel converters. They are harmonically equivalent, with the best spectral performance being achieved when the nearest three space vector states are selected with the middle two vectors centered in each half carrier switching interval, [11]. This strategy is known as centered space vector PWM. The vector centering can be done either implicitly using phase disposition carrier PWM with a common mode offset added to the phase leg reference waveforms, or explicitly using a space vector implementation.

Fig. 3 shows what the space vector $(\alpha, \beta)$ plane looks like for a three-level converter. For this converter, voltage vectors are divided in four groups: zero vectors, $V_{0}$; small vectors $\left(V_{1}\right.$ and $V_{4}$ in the first $60^{\circ}$ sector); medium vectors, $V_{3}$; and large vectors $\left(V_{2}\right.$ and $V_{5}$ in the first sector).

Similarly to the SPWM or THPWM, space vector modulation is used to approximate a desirable voltage vector by assigning dwell times to its surrounding state vectors. As shown in Fig. $4, v_{1}, v_{2}$ and $v_{3}$ are used to approximate the desired voltage vector $v_{s}$ in a control cycle $T_{s}$.

The dwell times in the first half of the first $60^{\circ}$ sector are calculated according to (1) and (2) 


$$
\begin{gathered}
T_{s}=T_{1}+T_{2}+T_{3} \\
v_{s} T_{s}=v_{1} T_{1}+v_{2} T_{2}+v_{3} T_{3}
\end{gathered}
$$

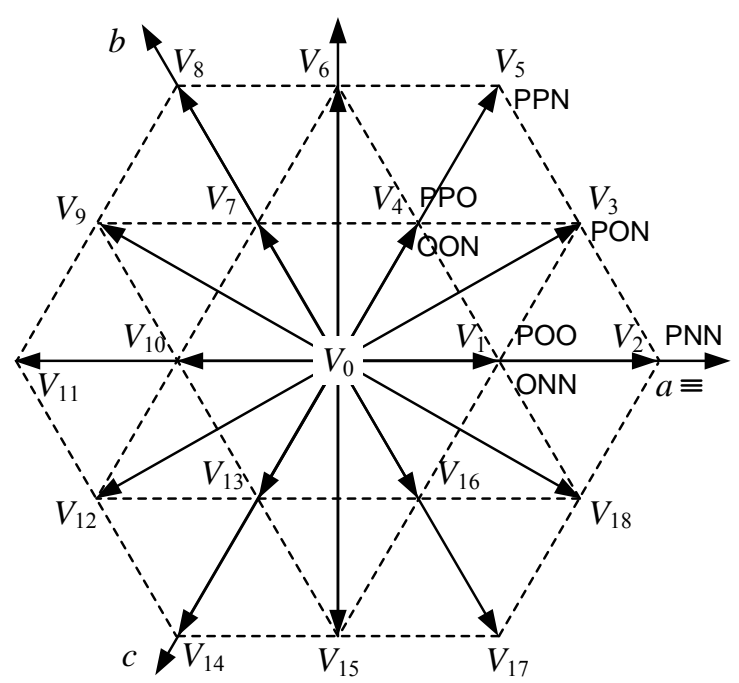

Fig. 3. Voltage vectors in the three-level NPC converter showing redundant small vectors in the first $60^{\circ}$ sector.

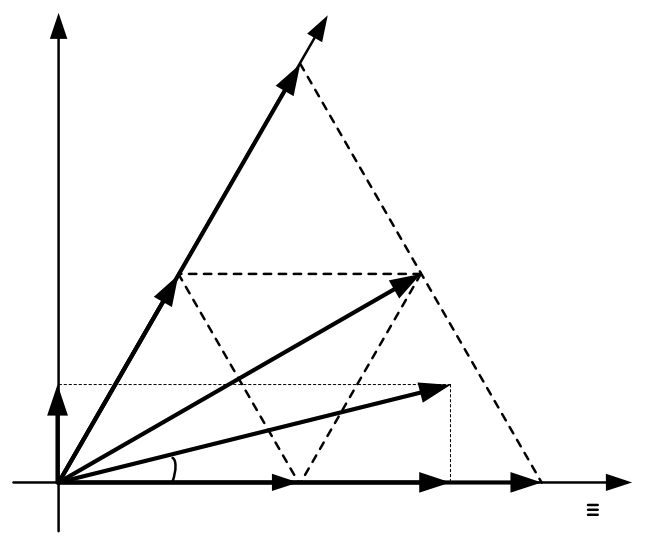

Fig. 4. Space vector modulation principle using the three nearest vectors in the first $60^{\circ}$ sector.

Solving the above two equations for individual dwell time for each surrounding vector leads to

$$
\left[\begin{array}{c}
T_{1} / T_{s} \\
T_{2} / T_{s} \\
T_{3} / T_{s}
\end{array}\right]=\left[\begin{array}{ccc}
v_{1 \alpha} & v_{2 \alpha} & v_{3 \alpha} \\
v_{1 \beta} & v_{2 \beta} & v_{3 \beta} \\
1 & 1 & 1
\end{array}\right] \cdot\left[\begin{array}{c}
v_{s \alpha} \\
v_{s \beta} \\
1
\end{array}\right]
$$

where $T_{1} / T_{s}, T_{2} / T_{s}$, and $T_{3} / T_{s}$, are the per unit times for each surrounding vectors. The voltages are expressed on their $(\alpha, \beta)$ reference frame.

The space vector modulation for more than three-level converters is very complex due to the high number of space vectors and redundant switching states, [11]. The use of Cartesian coordinates has two difficulties for more than three levels converters: 1 - it is very time consuming to determine which triangle the end point of the reference vector falls into; and 2- the equations for the calculation of dwell times usually change with the triangle that $V_{s}$ is in. To solve these problems, the Cartesian coordinate system can be transformed to a $60^{\circ}$ coordinate system, with $(g, h)$ axes, [12]. In the three-level system there is no such complexity, so this work will use the $(\alpha, \beta)$ coordinate system.

\section{Neutral Point Balancing}

The multilevel structure has an inherent problem of midpoint balance because the midpoints are used as power sources, when the midpoints are not independent power sources. As the level increases, the problem becomes more complicated. Therefore, the neutral point balance of the three-level case is easiest to handle. The direct reason for the unbalance is due to the current flow from/to the Neutral Point (NP).

Under normal operation the mean current drawn from the neutral point over a modulation cycle is zero and the midpoint point potential remains constant. Transient conditions at the load and AC mains level, imperfect symmetry in switching patterns, for instance due to deadtimes, are present in practical applications. These conditions will lead to drift of the neutral point potential to undesirable values, which result in asymmetrical load voltages and additional stress in the converter semiconductors. Therefore, an active copol alternative should be used in order to obtain zero NP p tential, [11].

The neutral point voltage balancing problem of threelevel NPC converters has been widely recognized and studied, [11], [13]. Various strategies have been presented, and successful operation has been demonstrated with a DC link voltage balance maintained for $N$-level converters, [14].

\section{A. Carrier PWM with DC Offset}

PWM generation using multicarrier techniques produces a balanced switching pattern, and the neutral point will remain constant unless the load becomes unbalanced. Additionally, as the output voltage magnitude and the load current magnitude increase, and the power factor approaches zero, the neutral point potential fluctuation increases. In particular, the neutral point potential fluctuation is maximum when operating at the maximum output voltage with near-zero power factor, [12].

Essentially, the carrier PWM methods control the NP voltage adding a zero sequence signal to all the three phase modulation waves, [11], [13]. The addition of the same DC offset to the three modulation waves does not change the effective line-to-line voltage, but changes the effective current path through the capacitor bank.

As already noticed, the addition of a homopolar component is valuable as it increases the converter gain: the optimal DC bus utilization (maximum gain) can only be reached by using the homopolar component and allows a $15 \%$ voltage additional gain. Also, it allows the reduction of switching losses, [15]. The homopolar component can be seen as a degree of freedom that allows the control of the neutral point voltage.

With reference to Fig. 1, the DC voltage unbalance is given by: 


$$
\Delta v_{C}=v_{C p}-v_{C n}
$$

Assuming equal capacitors, the relation between the neutral point voltage and the neutral current is:

$$
i_{0}=C \frac{d v_{C p}}{d t}-C \frac{d v_{C n}}{d t}=C \frac{d \Delta v_{C}}{d t}
$$

The mean value of the neutral current during one switching period $T_{s}$ can be determined from the phase currents $\left(i_{a}, i_{b}, i_{c}\right)$ and the switching functions of the modulator according to (6).

$$
<I_{0}>\left.\right|_{T_{S}}=\left|S_{a 0}\right| \cdot i_{a}+\left|S_{b 0}\right| \cdot i_{b}+\left|S_{c 0}\right| \cdot i_{c}
$$

If a homopolar component is added to the switching functions (7) is obtained

$$
<I_{0}>\left.\right|_{T_{S}}=\left|S_{a n}+S_{n 0}\right| \cdot i_{a}+\left|S_{b n}+S_{n 0}\right| \cdot i_{b}+\left|S_{c n}+S_{n 0}\right| \cdot i_{c}
$$

being $S_{i n}, i=a, b, c$, the reference load switching functions and $S_{n 0}$ the added homopolar component.

Since the voltage references as well as the phase currents are imposed and constant during a switching period, it appears clearly that the homopolar component $S_{n 0}$ has a direct influence on $i_{0}$ and thereby on $\Delta v_{c}$.

\section{B. Using Redundant States in SVPWM}

Maintaining voltage balance on the capacitors can be accomplished through selection of the redundant states. Redundant switching states create the same output voltages, but yield different capacitor currents. Therefore, capacitor voltage balancing through redundant state selection is a straightforward matter of selecting between the redundant states based on which capacitor is overcharged with respect to the other and the direction of the phase currents. Essentially, the same that is made under carrier based PWM control.

The medium voltage vectors do not flow current when the loads are balanced and resistive only. Otherwise, they affect the midpoint voltage, because the capacitors are charged or discharged according to the unbalanced and/or non-resistive load conditions. This condition does not allow for the charge balance, because there is no other vector to compensate it; the small vectors must be used. Fig. 5 shows the space vector control signals for the NPC converter in the first half of the first $60^{\circ}$ sector. Even at a high modulation index, the redundant states POO and ONN must be used in order to guarantee NP voltage control.

The majority of the NP voltage balancing schemes used in SVPWM relies on some form of manipulation of small vectors in a pair, where the relative duration of positive and negative small vectors in a pair is usually adjusted in order to compensate for the error in the neutral point, [11].

The influence of the small redundant vectors on the neutral point potential is illustrated in Fig. 6. As the figure suggests, when selecting a pair of redundant vectors, $(\mathrm{POO}, \mathrm{ONN})$ in the figure, the redundancy ratio must be carefully selected in order to control the average neutral point current flow over the PWM cycle.

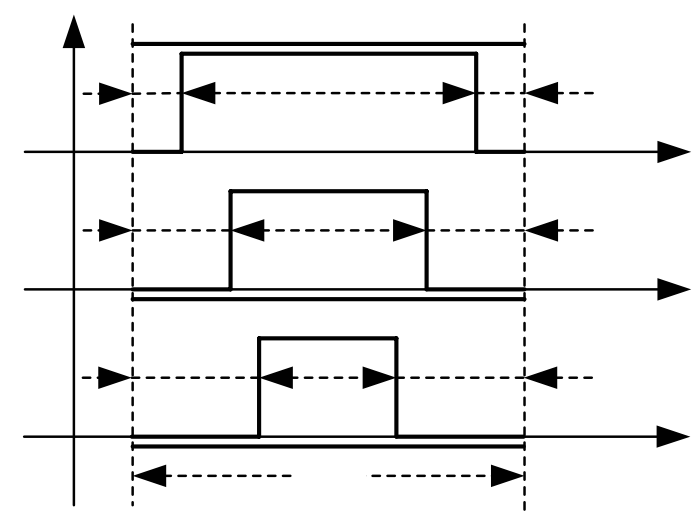

Fig. 5. SVPWM control signals for the NPC converter in the first half of the first $60^{\circ}$ sector showing redundant states POO and ONN (small vectors).
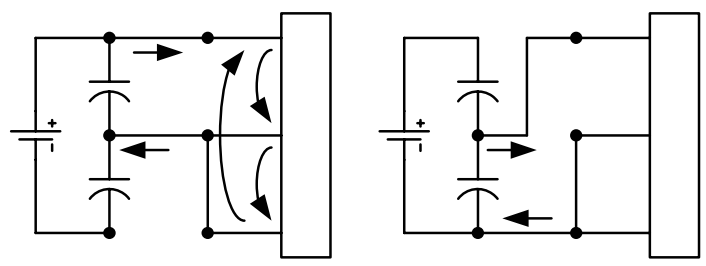

Fig. 6. One set of redundant states (POO and $\mathrm{ONN}$ ) with different neutral point currents and charging/discharging paths.

Obviously, the increment or decrement in the neutral voltage depends on the selected vector, the load current, the pulse duration, and the employed capacitor.

There seems to be equivalence in the NP balance control mechanism between carrier and space vector based PWM methods, [11]. In carrier PWM, all the control schemes are based on the use of output zero sequence voltage. Similarly, all the NP control methods based on space vectors use some manipulation of the redundant small vectors. The difference between the phase voltages of two small vectors in a pair is, in fact, the zero sequence voltage, as illustrated in Fig. 6.

\section{Results}

In order to show the general equivalence between carrierbased modulation and space vector modulation a laboratory prototype of a 4-kW three-phase NPC converter has been built using three SK50MLI066 modules from Semikron. The complete set-up is shown in Fig. 7. The DC voltage was set to $250 \mathrm{~V}$, the switching frequency has a value of $1.6 \mathrm{kHz}$, and a $2-\mathrm{HP}$ induction motor has been connected to the system as a load. The control strategies were implemented in a DSP board based on TMS320F2812 from Texas Instruments.

\section{A. Simulation Results}

Two essays are presented: line-to-line output voltage spectrum and DC capacitors voltage and current. Fig. 8 
and 9 show the line-to-line output voltage spectrum with $f_{o}=50 \mathrm{~Hz}$ and a modulation index of 0.95 for both methods, THPWM and SVPWM, respectively.

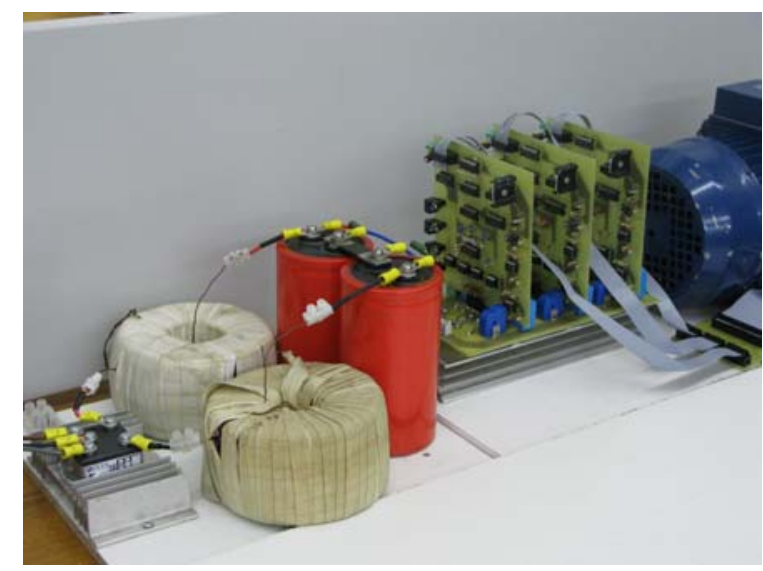

Fig. 7. Laboratory set-up for testing the 4-kW NPC converter.

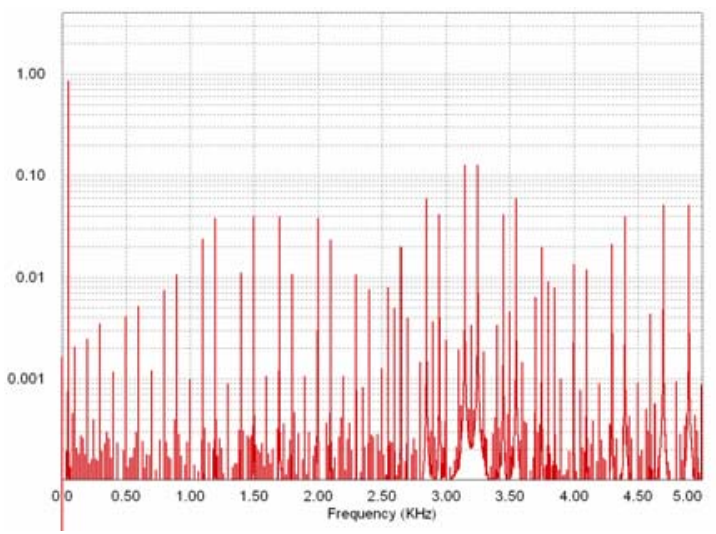

Fig. 8. THPWM line-to-line output voltage spectrum.

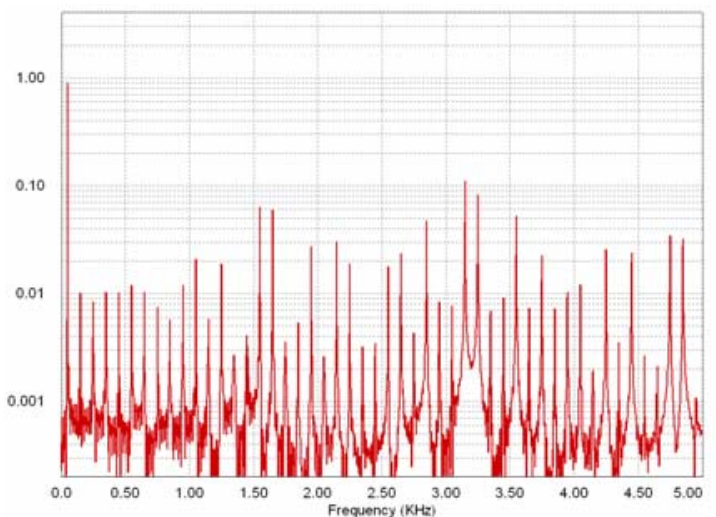

Fig. 9. SVPWM line-to-line output voltage spectrum.

Neglecting some minor spectral lines, essentially due to measurement noise, it can be concluded that the two methods have a very close spectrum.

Fig. 10 and 11 show the two capacitor voltages and the neutral-point injected current for both modulation methods: THPWM and SVPWM, respectively. [An offset voltage was used for comparative analysis.]

The same conclusion can be associated with the two methods: for the same conditions, the DC voltage behaviour is quite similar. It can be noticed a smaller ripple voltage with the SVPWM method associated with a slightly different switching behaviour.

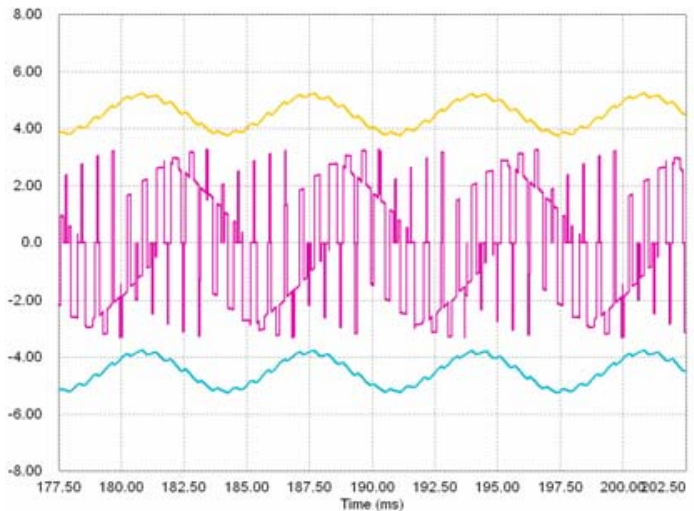

Fig. 10. Ripple voltage in the two capacitors $(2 \mathrm{~V} / \mathrm{div})$ and neutral-point input current $(2 \mathrm{~A} / \mathrm{div})$ for the THPWM method.

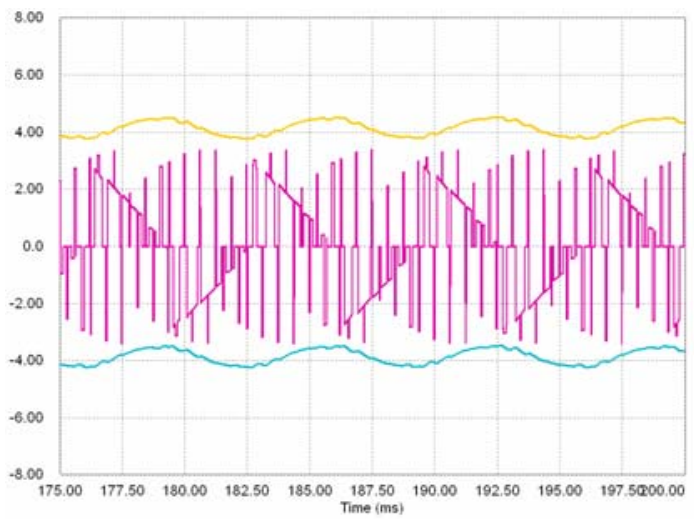

Fig. 11. Ripple voltage in the two capacitors $(2 \mathrm{~V} / \mathrm{div})$ and neutral-point input current $(2 \mathrm{~A} / \mathrm{div})$ for the SVPWM method.

\section{B. Experimental Results}

The briefly described prototype was used to obtain demonstrative experimental results. The same conditions were applied for the line-to-line voltage spectrum and for the DC capacitor ripple voltage; the respective spectrum and waveforms are shown in Fig. 12 and 13 for the output voltage and in Fig. 14 and 15 for the capacitor voltage.

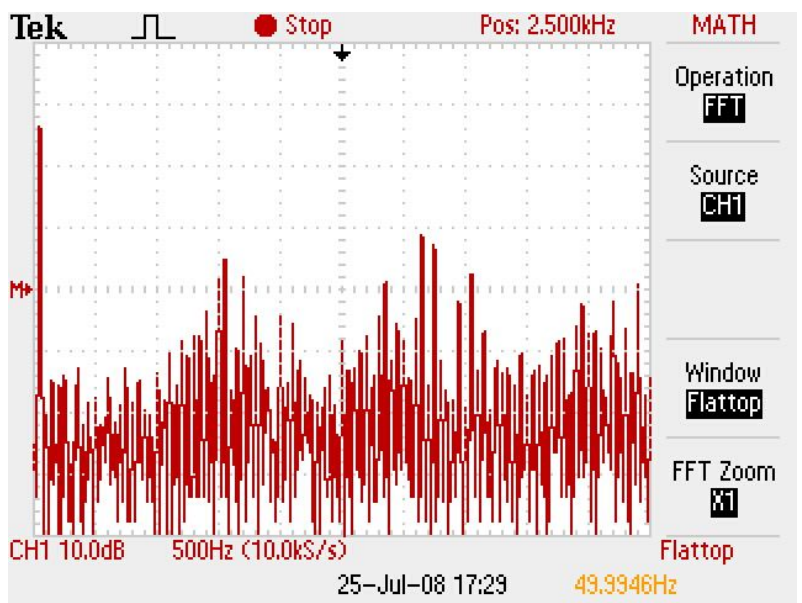

Fig. 12. THPWM line-to-line output voltage spectrum. 


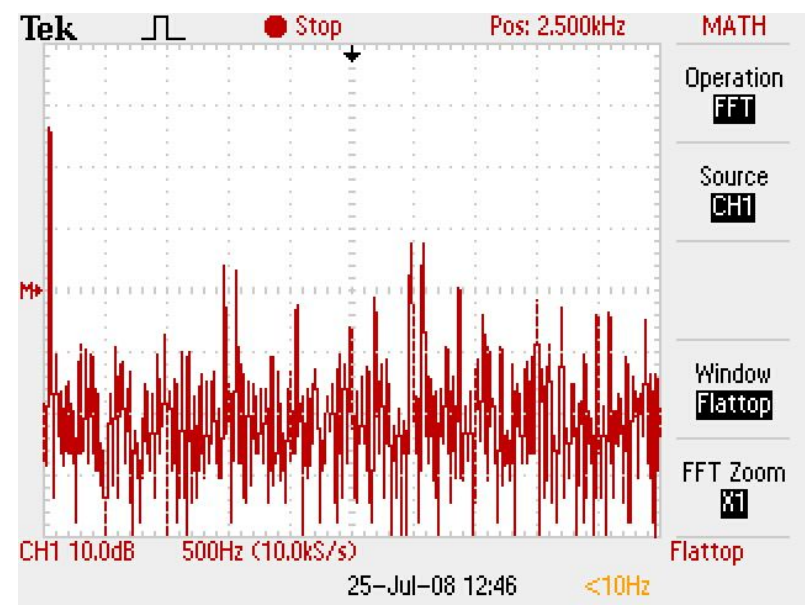

Fig. 13. SVPWM line-to-line output voltage spectrum.

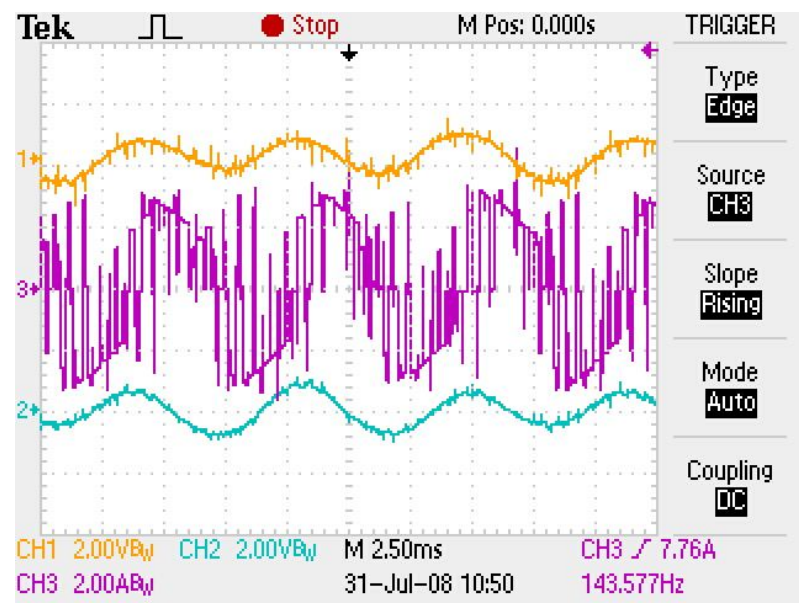

Fig. 14. Ripple voltage in the two capacitors and neutral-point input current for the THPWM method.

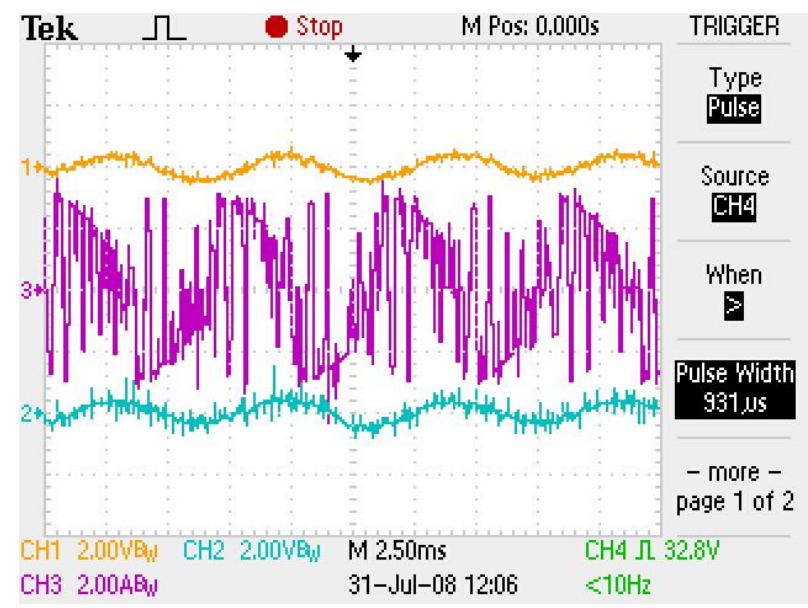

Fig. 15. Ripple voltage in the two capacitors and neutral-point input current for the SVPWM method.

The same comments and conclusions made with respect to the simulation results can be applied to the experimental ones: the main operational characteristics of the two methods are quite similar; also, but not shown in this paper, neutral point balancing is obtained with the same methodology, controlling the redundant vectors.

\section{Conclusions}

The three-phase NPC converter is a powerful and reliable converter for the medium-voltage high-power range. Its inner control system can be based on carrier or space vector PWM. The paper presents simulation and experimental results regarding its main performance issues namely output voltage spectrum and neutral point voltage balancing through DC capacitor current control. These results show that there is an equivalent performance between the two modulation strategies.

\section{References}

[1] B. Wu, High Power Converters and AC Drives, Wiley-IEEE Press, Hoboken, New Jersey, 2006.

[2] L. M. Tolbert, F. Z. Peng, and T. G. Habetler, "Multilevel converters for large electric drives", IEEE Trans. Industry Applications, vol. 35, no. 1, pp. 36-44, Jan./Feb. 1999.

[3] D. Soto and T. C. Green, "A comparison of high-power converter topologies for the implementation of FACTS controllers", IEEE Trans. Industrial Electronics, vol. 49, no. 5, pp. 1072-1080, Oct. 2002.

[4] A. Hodder, J.-J. Simond, and A. Schwery, "Double-fed asynchronous motor-generator equipped with a three-level VSI cascade", IEEE Industry Applications Magazine, vol. 14, no. 2, pp. 32-39, Mar./Apr. 2008

[5] J. Rodríguez, J.-S. Lai, and F. Z. Peng, "Multilevel converters: a survey of topologies, controls, and applications", IEEE Trans. Industrial Electronics, vol. 49, no. 4, pp. 724-738, Aug. 2002.

[6] A. Nabae, I. Takahashi, and H. Akagi, "A new neutral-pointclamped PWM converter," IEEE Trans. Industry Applications, vol. 17 , pp. 518-523, Sept. 1981.

[7] G. Carrara, S. Gardella, M. Marchesoni, R. Salutari, and G. Sciutto, "A new multilevel PWM method: a theoretical analysis", IEEE Trans. Power Electronics, vol. 7, no. 3, pp. 497-505, Jul.

[8] D. G. Holmes and T. A. Lipo, Pulse Width Modulation for Power Converters: Principles and Practice, IEEE Press, Piscataway, NJ, 2003.

[9] W. Yao, H. Hu, and Z. Lu, "Comparisons of space vector modulation and carrier-based modulation of multilevel converter", IEEE Trans. Industrial Electronics, vol. 23, no. 1, pp. 45-51, Jan. 2008.

[10] B. P. McGrath, D. G. Holmes, and T. Lipo, "Optimized space vector switching sequences for multilevel converters", IEEE Trans. Power Electronics, vol. 18, no. 6, pp. 1293-1301, Nov. 2003.

[11] N. Celanovic and D. Boroyevich, "A comprehensive study of neutral point voltage balancing problem in three-level neutralpoint-clamped voltage source PWM converters", IEEE Trans. Power Electronics, vol. 15, no. 2, pp. 242-249, Mar. 2000.

[12] S. Wei, B. Wu, F. Li, and C. Liu, "A general space vector PWM control algorithm for multilevel converters", in Proc. Applied Power Electronics Conference and Exposition (APEC'03), vol. 1, pp. 562-568, 2003.

[13] S. Ogasawara and H. Akagi, "Analysis of variation of neutral point potential in neutral-point-clamped voltage source PWM converters," in Proc. IEEE Ind. Applicat. Soc. Conf. Rec., 1993, pp. $965-970$.

[14] G. Sinha and T. A. Lipo, "A four-level converter based drive with a passive front end," IEEE Trans. Power Electronics, vol. 15, no. 2, pp. 285-294, Mar. 2000.

[15] A. Videt, P. Le Moigne, N. Idir, P. Baudesson, and X. Cimetière, "A new carrier-based PWM providing common-mode-current reduction and DC-bus balancing for three-level converters", IEEE Trans. Industrial Electronics, vol. 54, no. 6, pp. 3001-3011, Dec. 2007. 